\title{
Power Maximized and Anti-Saturation Power Conditioning Circuit for Current Transformer Harvester on Overhead Lines
}

\author{
Zhongnan Qian ${ }^{1}$, Jiande $\mathrm{Wu}^{1 *}$, Xiangning $\mathrm{He}^{1}$, Zhengyu Lin $^{2}$ \\ ${ }^{1}$ College of Electrical Engineering, Zhejiang University, Hangzhou, China \\ ${ }^{2}$ Department of Electrical Engineering and Electronic, Aston University, Birmingham, United Kingdom \\ *eewjd@zju.edu.cn
}

\begin{abstract}
The current transformer (CT) harvester is an effective and efficient solution due to its higher reliability and power density compared to other techniques. However, the current of overhead conductor fluctuates from tens of to thousands of amperes, which brings two challenges for the CT harvester design. First, the startup current, above which the harvester can independently power the monitoring devices, should be as low as possible, so that the battery capacity can be reduced; secondly, the magnetic core should be ensured unsaturated in high current condition. This paper proposes a power conditioning circuit with comprehensive control to maximize the output power and prevent the core from saturation. $A$ prototype that can deliver $22.5 \mathrm{~W}$ power with $200 \mathrm{~A}$ is designed, and a control strategy based on the finite-state machine is implemented. Experimental results show that the startup current for $\mathbf{2} \mathbf{W}$ load is about $30 \mathrm{~A}$, and the core power density at $60 \mathrm{~A}$ is $45.96 \mathrm{~mW} / \mathrm{cm}^{3}$, both of which are markedly improved compared to the reported results of the same condition.
\end{abstract}

\section{Introduction}

Condition monitoring (CM) for overhead lines (OHLs), such as temperature monitoring, fault detection and sag measurement, plays an essential role in the electrical power transmission, helping to reduce maintenance costs and improve the transmission reliability. The real-time thermal rating (RTTR) can significantly improve the transmission capacity while more monitoring and communication devices are required [1]. It is a challenge to power these devices on OHL because the power supply should be stable without deadtime and reliable in the harsh environment. Energy harvesting that overcomes the battery replacement issue has become an effective solution. Harvesters based on different energy sources have been reported, including vibration (piezoelectric [2], electromagnetic [3] and electrostatic [4]), electromagnetic coupling (electric coupling [5], and magnetic coupling [6]-[18]), thermal [19], solar [20] and wind [21]. Solar and wind harvesters heavily rely on the weather condition, so the energy storage element is bulky for a continuous power supply. Thermoelectric generators are unsuitable for OHL because of the wide temperature swing between the power line and ambient environment. An electric coupling harvester with power conditioning circuit has been presented in [5]. It has been demonstrated that the harvesting tube can achieve $370 \mathrm{~mW}$ on $150 \mathrm{kV}$ ac lines. The tube diameter is $30 \mathrm{~cm}$, and the length is $55 \mathrm{~cm}$.

Besides, the magnetic coupling harvester using the conductor current has been studied. According to the placed position, two types of magnetic coupling harvester are termed in [17]: enclosing-line current transformer (CT) harvesters [6]-[16] and free-standing harvesters [17], [18]. The CT harvester is applied in the scenario where CT is allowed to be clamped around the power line and more power is demanded, whereas the free-standing harvester is appropriate for the scenario where CT cannot be installed and less power is required. Therefore, the CT harvester is more suitable for OHL monitoring.

Since the current of OHL fluctuates from tens of to thousands of Amps, the CT harvester design is quite different from the low-current-range cases (from several Amps to tens of Amps) [6]-[9]. The CT harvester for OHL needs to maximize the power in low current condition for the reduction of backup batteries. Meanwhile, the saturation of magnetic core should be avoided when the current becomes high. Since the magnetostriction effect in saturation is serious and the split core will produce noise and physical vibration, which will result in the installation damage and insulation deterioration [22]. The voltage distortion caused by saturation will produce high-order harmonics and do harm to the grid and the associated circuits [23]. Thus, the air gap of magnetic core is employed for extending the unsaturated working range. However, too large air gap will degrade the power harvest in low current condition. An overvoltage protective unit consisting of a solid-state relay and a dissipative resistor in [10] is proposed to prevent the core saturation. However, the dissipative resistor will increase the self-dissipation of power supply. In [11], two secondary coils are implemented, and they are toggled when the primary current changes, so as to maximize the power in low current condition and to prevent the saturation in high current condition. But the CT design is more complicated.

Power electronic circuits with specific control methods are widely used for voltage conversion and power regulation in both ac and dc networks [24]. This paper proposes a power conditioning circuit with comprehensive control for CT harvester. The advantages of the proposed method are:

1) The harvested power is maximized. From a novel viewpoint, the buck-boost converter is implemented for impedance matching to achieve maximum power transfer. The power maximization in low current condition is beneficial to reduce the capacity of backup battery.

2) Short-circuit angle regulation is proposed to prevent the core saturation, which is realised by a semi-controlled active rectifier (S-CAR). This method will not result in extra self-dissipation like the dissipative resistor method.

The organization of this paper is: in Section 2, the power harvest of CT without conditioning circuit is analysed; in Section 3, the power maximizing circuit is presented and the 


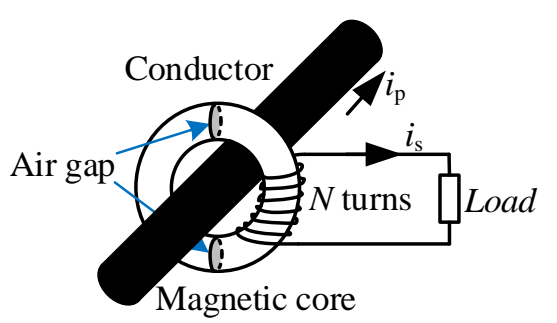

Fig. 1. CT harvester

impedance matching method is analysed; in Section 4, the short-circuit angle regulation for anti-saturation is proposed and analysed; in Section 5, a control strategy based on finite-state machine is designed for the variable primary current; in Section 6, experimental results are presented to verify these methods; in Section 7, the conclusions are given.

\section{CT Harvesting Analysis}

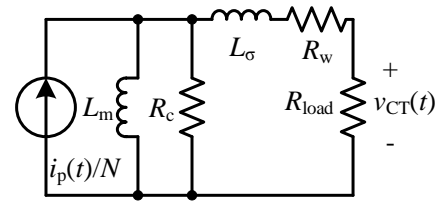

(a)

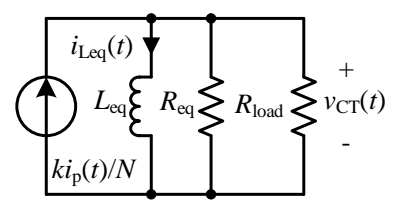

(b)
Fig. 2. CT circuit model

(a) General model, (b) Norton equivalent model

For the CT harvester on OHL, as shown in Fig. 1, high saturation flux density $B_{\text {sat }}$ and high permeability $\mu_{\mathrm{r}}$ of the magnetic core are required. A non-oriented electrical steel toroidal core is used in this paper. The core parameters are listed in Table 1. For the core with air gap, the equivalent relative permeability is given by [10].

$$
\mu_{\mathrm{eq}}=\frac{\mu_{\mathrm{r}}}{1+\mu_{\mathrm{r}} \frac{a}{l}}
$$

Generally, the CT can be modelled by the magnetizing inductance $L_{\mathrm{m}}$, leak inductance $L_{\sigma}$, wire loss resistance $R_{\mathrm{W}}$ and core loss resistance $R_{\mathrm{c}}$ [6], as shown in Fig. 2(a). This circuit model can be further simplified by Norton Theorem, as shown in Fig. 2(b). The equivalent inductance $L_{\mathrm{eq}}$, equivalent loss resistance $R_{\text {eq }}$ and coefficient $k$ of the current source are derived as

$$
\begin{aligned}
R_{\mathrm{eq}} & =\frac{\left(R_{\mathrm{c}} R_{\mathrm{w}}-\omega^{2} L_{\mathrm{m}} L_{\sigma}\right)^{2}+\omega^{2}\left(L_{\mathrm{m}} R_{\mathrm{c}}+L_{\mathrm{m}} R_{\mathrm{w}}+L_{\sigma} R_{\mathrm{c}}\right)^{2}}{R_{\mathrm{c}}{ }^{2} R_{\mathrm{w}}+\omega^{2} L_{\mathrm{m}}{ }^{2}\left(R_{\mathrm{c}}+R_{\mathrm{w}}\right)} \\
L_{\mathrm{eq}} & =\frac{\left(R_{\mathrm{c}} R_{\mathrm{w}}-\omega^{2} L_{\mathrm{m}} L_{\sigma}\right)^{2}+\omega^{2}\left(L_{\mathrm{m}} R_{\mathrm{c}}+L_{\mathrm{m}} R_{\mathrm{w}}+L_{\sigma} R_{\mathrm{c}}\right)^{2}}{\omega^{4} L_{\mathrm{m}}{ }^{2} L_{\sigma}+\omega^{2}\left(L_{\mathrm{m}}+L_{\sigma}\right) R_{\mathrm{c}}^{2}} \\
k & =\frac{j \omega L_{\mathrm{m}} R_{\mathrm{c}}}{\left(R_{\mathrm{c}} R_{\mathrm{w}}-\omega^{2} L_{\mathrm{m}} L_{\sigma}\right)+j \omega\left(L_{\mathrm{m}} R_{\mathrm{c}}+L_{\sigma} R_{\mathrm{c}}+L_{\mathrm{m}} R_{\mathrm{w}}\right)}
\end{aligned}
$$

The theoretical relationships between the two circuit models are described by (2), (3) and (4). However, it is unnecessary to derive the Norton parameters from the general model parameters in the practical measurement. The Norton parameters can be directly measured by the methods which are commonly used to determine a Norton equivalent circuit, such as the method using open-circuit voltage and short-circuit current, and the method of adding a test voltage source. The measurement process will be presented in Section 6. The Norton parameters of the adopted CT are measured and listed in Table 2.

\begin{tabular}{|c|c|c|}
\hline Symbol & Parameter & Value \\
\hline$N$ & Secondary turns & 600 \\
\hline$L_{\mathrm{eq}}$ & Norton Equivalent inductance & $460 \mathrm{mH}$ \\
\hline$R_{\mathrm{eq}}$ & Norton Equivalent resistance & $3.65 \mathrm{k} \Omega$ \\
\hline$k$ & Current source coefficient & $\approx 1$ \\
\hline
\end{tabular}

Table 1 Magnetic core parameters

\begin{tabular}{llc}
\hline \hline \multirow{2}{*}{ Symbol } & \multicolumn{1}{c}{ Parameter } & Value \\
\cline { 3 - 3 } & \multicolumn{1}{c}{ height } & $30 \mathrm{~mm}$ \\
$r_{\text {in }}$ & inner radius & $35 \mathrm{~mm}$ \\
$r_{\text {out }}$ & outer radius & $50 \mathrm{~mm}$ \\
$r_{\text {mid }}$ & middle radius & $42.5 \mathrm{~mm}$ \\
$A_{\text {eff }}$ & effective flux area & $443 \mathrm{~mm}^{2}$ \\
$a$ & total air gap length & $0.36 \mathrm{~mm}$ \\
$l$ & average length of magnetic path & $266.9 \mathrm{~mm}$ \\
$\mu_{\mathrm{r}}$ & relative permeability & 3960 \\
$B_{\text {sat }}$ & saturation flux density & $1.35 \mathrm{~T}$ \\
\hline \hline
\end{tabular}

Table 2 CT model parameters

Denote the primary current by $i_{\mathrm{p}}(t)=I_{\mathrm{p}} \sin (\omega t+\delta)$. The amplitude of $i_{\text {Leq }}(t)$ can be derived as

$$
I_{\text {Leq }}=\frac{k I_{\mathrm{p}}}{N \cdot \sqrt{\left(\frac{\omega L_{\mathrm{eq}}}{R_{\mathrm{eq}} / / R_{\mathrm{load}}}\right)^{2}+1}}
$$

The core operating point can be expressed by $I_{\text {Leq }}$.

$$
B_{\mathrm{op}} \approx \frac{\mu_{0} \mu_{\mathrm{eq}} N I_{\mathrm{Leq}}}{2 \pi r_{\text {mid }}}=\frac{\mu_{0} \mu_{\mathrm{eq}} k I_{\mathrm{p}}}{2 \pi r_{\text {mid }} \cdot \sqrt{\left(\frac{\omega L_{\mathrm{eq}}}{R_{\mathrm{eq}} / / R_{\mathrm{load}}}\right)^{2}+1}}
$$

Therefore, as the amplitude $I_{\mathrm{p}}$ or $R_{\text {load }}$ increases, the core

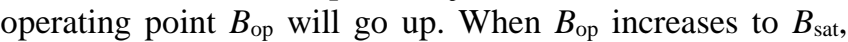
the CT output voltage at critical saturation is obtained.

$$
V_{\mathrm{CT}(\text { sat) }}=2 \pi f N A_{\text {eff }} B_{\text {sat }}
$$

Assume the core is working unsaturated, the CT output power is

$$
P_{\text {load }}=\frac{\left(V_{\text {CT }} / \sqrt{2}\right)^{2}}{R_{\text {load }}}=\frac{\left(\frac{k}{N} I_{\mathrm{p}}\right)^{2}}{2\left[\left(\frac{1}{R_{\text {eq }}}+\frac{1}{R_{\text {load }}}\right)^{2}+\left(\frac{1}{\omega L_{\text {eq }}}\right)^{2}\right] R_{\text {load }}}
$$

where $V_{\mathrm{CT}}$ is the amplitude of CT output voltage $v_{\mathrm{CT}}$. The maximum power transfer can be achieved when the load equals

$$
R_{\text {load }}=\frac{1}{\sqrt{\left(\frac{1}{R_{\text {eq }}}\right)^{2}+\left(\frac{1}{\omega L_{\text {eq }}}\right)^{2}}}
$$

The maximum CT output power is

$$
P_{\text {load(max })}=\frac{R_{\mathrm{eq}}}{4\left(1+\sqrt{1+Q^{2}}\right)}\left(\frac{k}{N} I_{\mathrm{p}}\right)^{2}
$$

where $Q$ is CT quality factor, defined as $Q=R_{\mathrm{eq}} / \omega L_{\mathrm{eq}}$.

\section{Power maximizing circuit}

\subsection{Reactive power compensation}

From Fig. 2(b), it can be seen that the equivalent inductor will shunt the source current and generate reactive power, which degrades the active power output. A parallel resonant capacitor $C_{\mathrm{r}}$ can be used to compensate the reactive current and improve the active power output, as shown in Fig. 3. The compensation capacitance is calculated by 


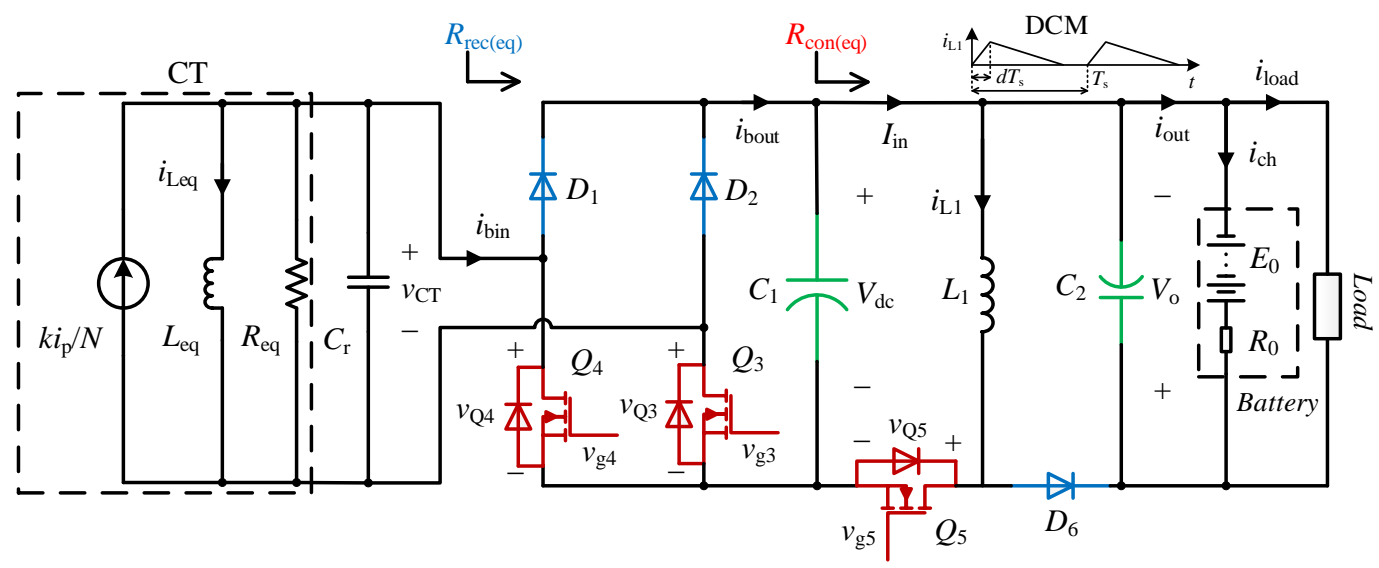

Fig. 3. Schematic of proposed power conditioning circuit

$$
C_{\mathrm{r}}=1 /\left(\omega_{\mathrm{r}}^{2} L_{\mathrm{eq}}\right)
$$

The output power of compensated CT can be derived as

$$
P_{\text {load }}=\frac{\left(\frac{k}{N} I_{\mathrm{p}}\right)^{2}}{2\left(\frac{1}{R_{\text {eq }}}+\frac{1}{R_{\text {load }}}\right)^{2} R_{\text {load }}}
$$

When $R_{\text {load }}=R_{\text {eq }}$, the maximum power transfer of compensated CT is achieved.

$$
P_{\text {load(max) }}=\frac{R_{\text {eq }}}{8}\left(\frac{k}{N} I_{\mathrm{p}}\right)^{2}
$$

Comparing (10) and (13), the maximum output power with compensation is increased by the ratio $\kappa$.

$$
\kappa=\frac{\left(1+\sqrt{1+Q^{2}}\right)}{2} \approx \frac{1+Q}{2}
$$

where the CT quality factor $Q \gg 1$.

\subsection{Conditioning circuit with impedance matching}

For the compensated CT, the maximum power transfer happens when the load resistance $R_{\text {load }}$ equals the CT equivalent loss resistance $R_{\text {eq. }}$. In practice, the monitoring devices powered by CT is considered as a dynamic load, the impedance of which is varying in a wide range. The maximum power transfer is hardly achieved. However, the power maximization in low current condition is necessary considering the capacity limit of backup battery. Therefore, a conditioning circuit with impedance matching function is designed, as shown in Fig. 3. The conditioning circuit consists of a compensation capacitor, a semi-controlled active rectifier (S-CAR) and a buck-boost converter. In impedance matching, the S-CAR works as a normal fullwave rectifier.

As we know, the buck-boost converter is commonly used for dc voltage conversion. However, it can be also used for impedance matching when it works in discontinuous conduction mode (DCM). The reason is that the input impedance of DCM buck-boost converter is resistive and can be derived as

$$
R_{\mathrm{con}(\mathrm{eq})}=\frac{V_{\mathrm{dc}}}{I_{\mathrm{in}}}=\frac{V_{\mathrm{dc}}}{\frac{V_{\mathrm{dc}}}{2 L_{1}} d^{2} T_{s}}=\frac{2 L_{1} f_{\mathrm{s}}}{d^{2}}
$$

where $f_{\mathrm{s}}$ is the switching frequency and $d$ is the duty cycle of $Q_{5}$.

From (15), the input resistance of DCM buck-boost converter is independent of input voltage and load impedance. If $L_{1}$ and $f_{\mathrm{s}}$ are determined, the input resistance of buck-boost convert can be solely controlled by duty cycle $d$. On the other hand, it can be derived that the input resistance of the full-wave rectifier is half of its load, i.e.

$$
R_{\text {rec(eq) }}=\frac{1}{2} R_{\text {con(eq) }}
$$

Thus, the impedance matching, or maximum power transfer, can be achieved by a constant duty cycle $d_{\text {mpp }}$ and independent of the dynamic load. Since the maximum power transfer condition is $R_{\text {rec(eq) }}=R_{\text {eq }}$, the duty cycle of maximum power transfer can be derived as

$$
d_{\mathrm{mpp}}=\sqrt{\frac{L_{1} f_{\mathrm{s}}}{R_{\mathrm{eq}}}}
$$

Therefore, the harvester in low current condition can maintain the maximum power transfer by setting the duty cycle to $d_{\mathrm{mpp}}$ regardless of the load varying.

\section{Short-circuit angle regulation}

When the conditioning circuit works at $d_{\text {mpp }}$ and the primary current increases to the high current condition, the magnetic core will be saturated. The critical primary current for saturation can be calculated by

$$
I_{\mathrm{p}(\text { crit })}=\frac{2 N V_{\mathrm{CT}(\mathrm{sat})}}{k R_{\mathrm{eq}}}
$$

If the duty cycle $d$ is regulated to prevent saturation when

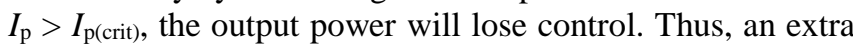
control variable called rectifier short-circuit angle is utilised to prevent the core saturation. Because the flux density is proportional to the integration of CT output voltage $\int_{0}^{t} v_{\mathrm{CT}} d t$, to control $v_{\mathrm{CT}}$ means to indirectly control the core

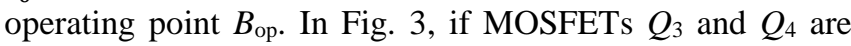
forced to turn on together, the CT output will be shortcircuited, that is $v_{\mathrm{CT}}=0$. If this happens in every period and lasts for a duration $T_{\text {short-circuit, }}$ the peak value of $v_{\mathrm{CT}}$ can be lowered. The core operating point $B_{\text {op }}$ is also lowered. The definition of short-circuit angle is given as

$$
\varphi=\frac{T_{\text {short-circuit }}}{T_{\text {period }}} \cdot 2 \pi
$$

The operating process of S-CAR with short-circuit angle are depicted in Fig. 4. It should be noted that MOSFETs $Q_{3}$ and $Q_{4}$ are also used as synchronous rectifiers to improve the rectifier efficiency. 


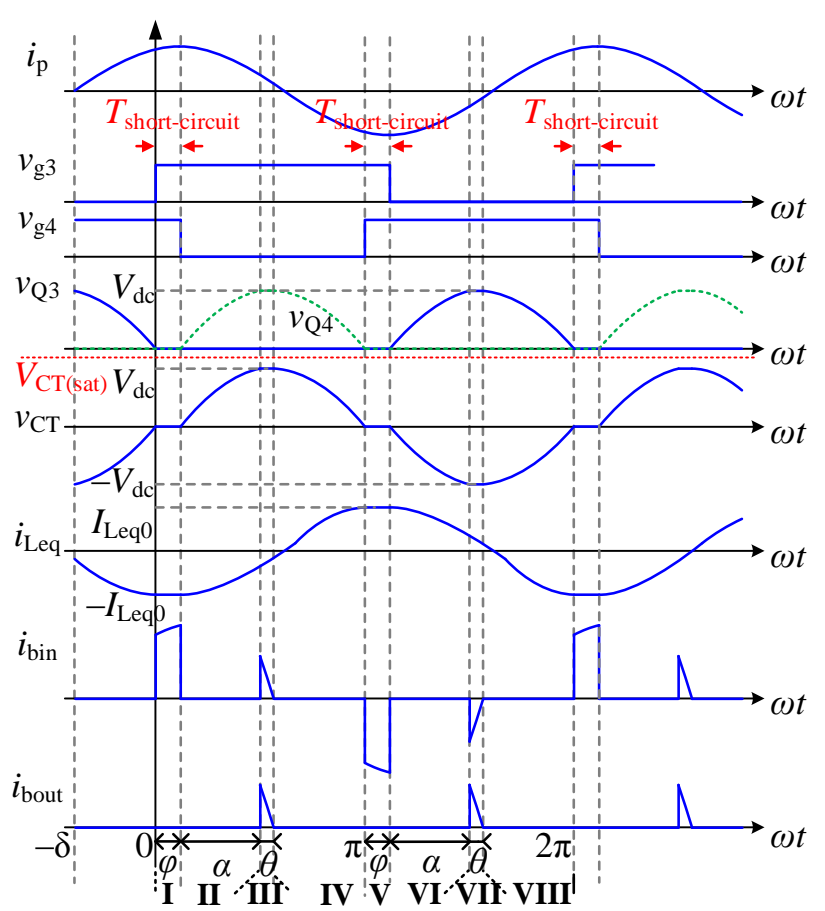

Fig. 4. Operating process of S-CAR with short-circuit angle

Interval $I[0, \varphi]: Q_{4}$ has been turned on as synchronous rectifier before $t=0$, so $v_{\mathrm{Q} 3}$ equals $-v_{\mathrm{CT}}$. When $v_{\mathrm{Q} 3}$ reaches zero from positive at $t=0, Q_{3}$ is turned on. Then the CT output is short-circuited. Thus, the CT output voltage and equivalent inductor current are

$$
\begin{gathered}
v_{\mathrm{CT}}(t)=0 \\
i_{\text {Leq }}(t)=-I_{\text {Leq } 0}
\end{gathered}
$$

Interval II $[\varphi, \varphi+\alpha]$ : At the end of short-circuit angle, $Q_{4}$ is turned off. Then the CT output voltage $v_{\mathrm{CT}}$ rises from zero to rectifier output voltage $V_{\mathrm{dc}}$. It should be noted that $V_{\mathrm{CT} \text { (sat) }}$ derived from (7) is the upper limit of $v_{\mathrm{CT}}$ for anti-saturation, which is depicted by red dashed line in Fig. 4. The inductor current and CT output voltage can be derived as

$$
\begin{gathered}
i_{\text {Leq }}(t) \approx A_{1} e^{-\alpha_{1}\left(t-\frac{\varphi}{\omega}\right)} \sin \left(\omega t+\varphi_{1}-\varphi\right)-\frac{Q k I_{\mathrm{p}}}{N} \cos (\omega t+\delta) \\
v_{\mathrm{CT}}(t)=L_{\mathrm{eq}} A_{1} e^{-\alpha_{1}\left(t-\frac{\varphi}{\omega}\right)}\left[\omega \cos \left(\omega t+\varphi_{1}-\varphi\right)-\alpha_{1} \sin \left(\omega t+\varphi_{1}-\varphi\right)\right] \\
+\frac{k I_{\mathrm{p}} R_{\mathrm{eq}}}{N} \sin (\omega t+\delta)
\end{gathered}
$$

where $\alpha_{1}=1 /\left(R_{\mathrm{eq}} C_{\mathrm{r}}\right)$.

Interval III $[\varphi+\alpha, \varphi+\alpha+\theta]: D_{1}$ is turned on. The CT output voltage $v_{\mathrm{CT}}$ is equal to the rectifier output voltage $V_{\mathrm{dc}}$, and $i_{\text {Leq }}$ increases linearly.

$$
\begin{aligned}
v_{\mathrm{CT}}(t)=V_{\mathrm{dc}} \\
i_{\mathrm{Leq}}(t)=A_{1} e^{-\frac{\alpha}{\omega} \alpha_{1}} \sin \left(\alpha+\varphi_{1}\right)-\frac{Q k I_{\mathrm{p}}}{N} \cos (\varphi+\alpha+\delta) \\
+\frac{V_{\mathrm{dc}}}{L_{\mathrm{eq}}}\left(t-\frac{\varphi+\alpha}{\omega}\right)
\end{aligned}
$$

Interval IV $[\varphi+\alpha+\theta, \pi]: D_{1}$ is turned off. Then $v_{\mathrm{CT}}$ decreases from $V_{\mathrm{dc}}$ to zero. The inductor current and CT output voltage have similar forms with Interval II.

$$
\begin{aligned}
i_{\text {Leq }}(t) \approx & A_{2} e^{-\alpha_{1}\left(t-\frac{\varphi+\alpha+\theta}{\omega}\right)} \sin \left[\omega t+\varphi_{2}-(\varphi+\alpha+\theta)\right] \\
& -\frac{Q k I_{\mathrm{p}}}{N} \cos (\omega t+\delta)
\end{aligned}
$$

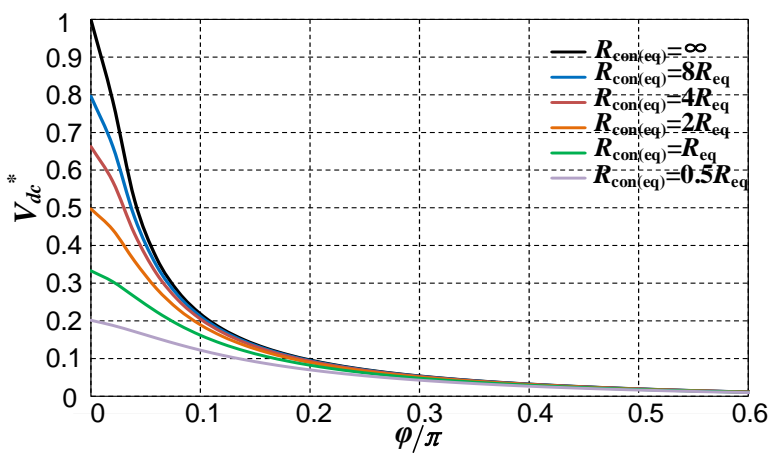

(a)

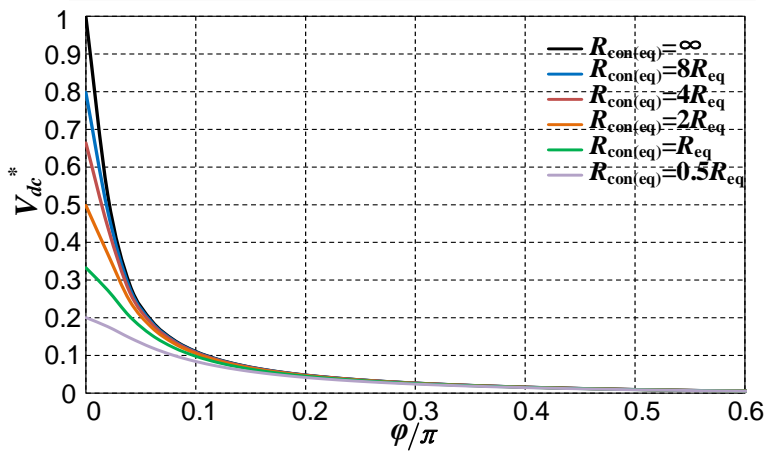

(b)

Fig. 5. CT output voltage peak $V_{d c} *$ versus short-circuit angle $\varphi$ : (a) $\mathrm{Q}=20,(\boldsymbol{b}) \mathrm{Q}=40$

$$
\begin{aligned}
& v_{\mathrm{CT}}(t)=L_{\mathrm{eq}} A_{2} e^{-\alpha_{1}\left(t-\frac{\varphi+\alpha+\theta}{\omega}\right)}\left\{\omega \cos \left[\omega t+\varphi_{2}-(\varphi+\alpha+\theta)\right]\right. \\
& \left.-\alpha_{1} \sin \left[\omega t+\varphi_{2}-(\varphi+\alpha+\theta)\right]\right\}+\frac{k I_{\mathrm{p}} R_{\mathrm{eq}}}{N} \sin (\omega t+\delta)
\end{aligned}
$$

Intervals $V-V I I I \quad[\pi, 2 \pi]$ : These four intervals are symmetric with Intervals $I-I V$.

From (20)-(27), nine continuity equations can be derived, which are listed in Appendices. Solving the simultaneous equations, the aforementioned coefficients $A_{1}, A_{2}, \varphi_{1}, \varphi_{2}, \delta$, $\alpha, \theta, I_{\text {Leq } 0}$ and $V_{\mathrm{dc}}$ can be determined. Since the continuity equations are transcendental equations, only the numerical solution is achieved by MAPLE.

The numerical relationship between CT output voltage peak $V_{\mathrm{dc}}$ and short-circuit angle $\varphi$ is depicted in Fig. 5, where $V_{\mathrm{dc}}{ }^{*}$ is the normalized value and $R_{\mathrm{con}(\mathrm{eq})}$ is the load resistance of rectifier. It can be seen that the falling of $V_{\mathrm{dc}}$ is fairly steep when $\varphi<0.1$. A higher quality factor $Q$ or a larger load resistance will make the falling steeper. It can be inferred that with a proper regulation of short-circuit angle, $V_{\mathrm{dc}}$ can be limited below $V_{\mathrm{CT}(\mathrm{sat})}$. Then the anti-saturation is achieved.

\section{Comprehensive control strategy}

For the real primary current with fluctuation, a comprehensive control strategy is designed. The finite-state machine programming is adopted to implement the control strategy on MCU. The harvester has three states, namely maximum output power state (MOP), limited output power state (LOP), and limited output voltage state (LOV), as shown in Fig. 6. The rectifier output voltage $V_{\mathrm{dc}}$ and the short-circuit angle $\varphi$ determines the state transition. Two reference voltages $V_{\mathrm{dc}(\mathrm{low})}$ and $V_{\mathrm{dc}(\text { high) }}$ are employed for state transition. Their values are quite close to the saturation voltage $V_{\mathrm{CT} \text { (sat) }}$ and comply with $V_{\mathrm{dc}(\text { low })}<V_{\mathrm{dc}(\text { high })}<V_{\mathrm{CT} \text { (sat). }}$. 


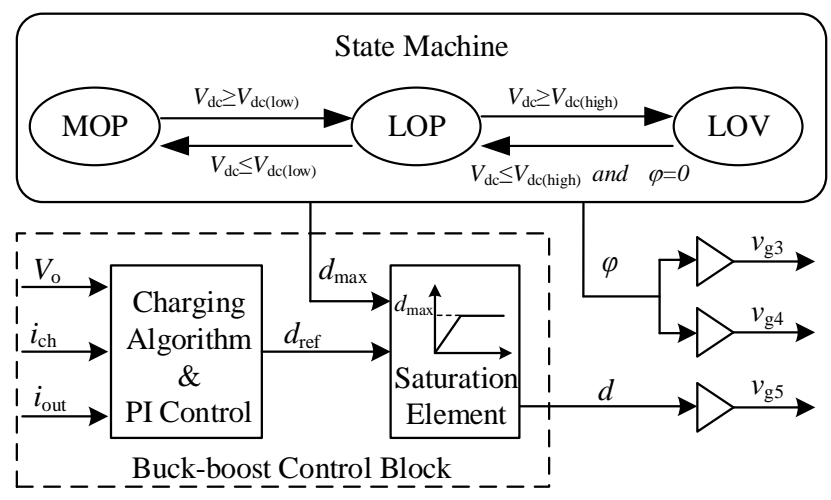

Fig. 6. Control diagram

The allowed maximum duty cycle $d_{\max }$ and the short-circuit angle $\varphi$ of the S-CAR are the output of state machine. It should be noted that the real duty cycle $d$ is regulated by the buck-boost control block while $d_{\max }$ only determines the upper limit. The buck-boost control block contains normal functions such as battery charging management and output voltage/current control, which will not be discussed in this paper.

MOP: The initial state of the state machine is the MOP state, which is for the low primary current condition. In this state, the short-circuit angle $\varphi$ is fixed to zero and $d_{\max }$ is set at $d_{\text {mpp. }}$. The buck-boost duty cycle $d$ is regulated in [0, $\left.d_{\mathrm{mpp}}\right]$. When the duty cycle is regulated to $d_{\mathrm{mpp}}$, the maximum power transfer is achieved. The rectifier output voltage $V_{\mathrm{dc}}$, however, is uncontrolled in MOP. The maximum output power of MOP can be derived from (13).

LOP: When the primary current increases and results in $V_{\mathrm{dc}} \geq V_{\mathrm{dc}(\text { low) }}$, the state machine changes from MOP state to LOP state. In LOP state, the short-circuit angle $\varphi$ remains zero and the maximum duty cycle $d_{\max }$ is designed to increase linear with $V_{\mathrm{dc}}$ as

$$
d_{\text {max }}=d_{\text {mpp }}+p_{\mathrm{d}}\left(V_{\mathrm{dc}}-V_{\mathrm{dc}(\text { low })}\right)
$$

where $p_{\mathrm{d}}$ is a constant coefficient. Thus, $V_{\mathrm{dc}}$ can be stabilized and keeps in the range $\left[V_{\mathrm{dc}(\text { low })}, V_{\mathrm{dc}(\text { high })}\right]$. Since $V_{\mathrm{dc}} \approx V_{\mathrm{CT} \text { (sat) }}$ in LOP, the magnetic core is working at critical saturation. Thus, the maximum output power can be calculated as

$$
P_{\max (\mathrm{LOP})}=\frac{k I_{\mathrm{p}} V_{\mathrm{CT}(\text { sat) }}}{2 N}-\frac{V_{\mathrm{CT}(\text { (sat) }}^{2}}{2 R_{\mathrm{eq}}}
$$

$L O V$ : When the primary current further increases and the required power remains small, the rectifier output voltage $V_{\mathrm{dc}}$ will be larger than $V_{\mathrm{dc}(\mathrm{high})}$. Then the state machine shifts to LOV state. In this state, the short-circuit angle $\varphi$ is regulated to keep $V_{\mathrm{dc}}$ equal to $V_{\mathrm{dc}(\mathrm{high})}$, and the maximum duty cycle $d_{\max }$ is fixed according to (28). The coordination of short-circuit angle and the duty cycle keeps the magnetic core working at the critical saturation.

In conclusion, the harvester output power is maximized by impedance matching in low current condition and by working at critical saturation in high current condition. Thus, the power maximization over the full current range is achieved. The maximum output capability is calculated by (30) with the approximation $V_{\mathrm{dc}(\text { low })} \approx V_{\mathrm{dc}(\text { high) }} \approx V_{\mathrm{CT} \text { (sat). }}$. The short-circuit angle is added in LOV state to prevent the core saturation, and meanwhile the critical saturated working is maintained, which can make full use of the core linear region and not result in much power reduction.

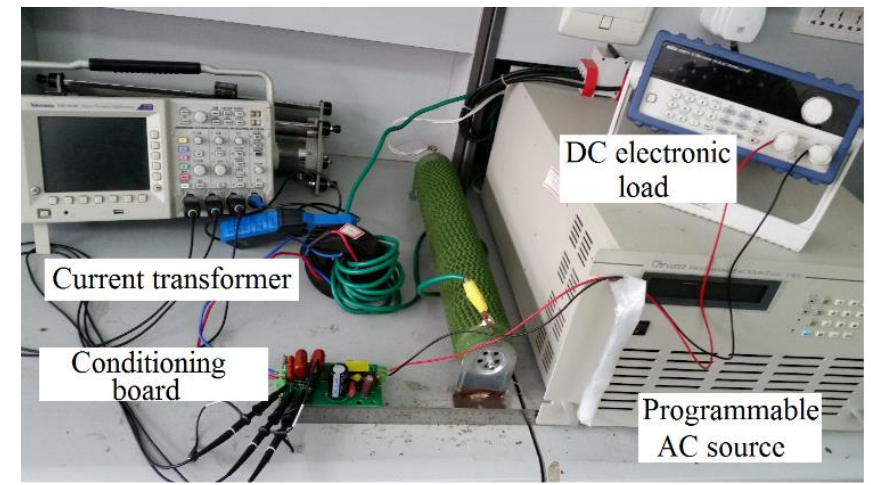

(a)

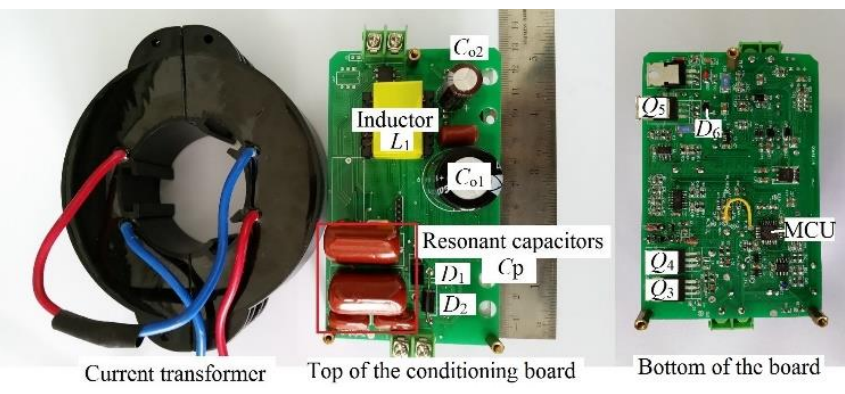

(b)

Fig. 7. Experimental setup

(a) Test bench, (b) CT and conditioning board

$$
P_{\mathrm{CT} \max }= \begin{cases}\frac{R_{\mathrm{eq}}}{8}\left(\frac{k}{N} I_{\mathrm{p}}\right)^{2} & \text { if } \frac{k I_{\mathrm{p}}}{N} \leq \frac{2 V_{\mathrm{CT}(\mathrm{sat})}}{R_{\mathrm{eq}}} \\ \frac{k I_{\mathrm{p}} V_{\mathrm{CT}(\text { sat) }}}{2 N}-\frac{V_{\mathrm{CT}(\mathrm{sat})}^{2}}{2 R_{\mathrm{eq}}} & \text { if } \frac{k I_{\mathrm{p}}}{N}>\frac{2 V_{\mathrm{CT}(\text { sat })}}{R_{\mathrm{eq}}}\end{cases}
$$

\section{Experimental verification}

A test bench was constructed and used to verify the proposed methods, as shown in Fig. 7(a). It included a programmable AC current source with $20 \mathrm{~A}(50 \mathrm{~Hz})$ maximum output, a sliding rheostat as AC resistor load, a DC electronic load and the CT. To achieve an equivalent of $200 \mathrm{~A}(50 \mathrm{~Hz})$ maximum primary current, 10 turns were wound on the CT primary side. A prototype of the conditioning circuit was designed, as shown in Fig. 7(b). A cortex M0 microcontroller STM32F051 was used to control the S-CAR and the buck-boost converter. The prototype parameters are listed in Table 3.

Table 3 Parameters of conditioning board

\begin{tabular}{llc}
\hline \hline Symbol & \multicolumn{1}{c}{ Parameter } & Value \\
\hline$C_{\mathrm{r}}$ & Compensation capacitor & $22 \mu \mathrm{F}$ \\
$C_{1}$ & Rectifier output capacitor & $470 \mu \mathrm{F}$ \\
$C_{2}$ & Buck-boost output capacitor & $220 \mu \mathrm{F}$ \\
$L_{1}$ & Buck-boost inductor & $1.09 \mathrm{mH}$ \\
$f_{\mathrm{s}}$ & Buck-boost switching frequency & $50 \mathrm{kHz}$ \\
$E_{0}$ & Battery voltage & $48 \mathrm{~V}$ \\
$D_{1} D_{2}$ & Rectifier diodes & $1 \mathrm{~N} 5408$ \\
$Q_{3} Q_{4} Q_{5}$ & MOSFETs & IRF640 \\
$D_{6}$ & Buck-boost diode & RS1G \\
\hline \hline
\end{tabular}

The measurement setups of the CT Norton parameters are illustrated in Fig. 8. The measurement process is as follows.

1) In Fig. 8(a), provide a primary current $\boldsymbol{I}_{\mathbf{p}}$ (including amplitude and phase) and short circuit the secondary side. Then the short-circuit current $\boldsymbol{I}_{\text {sc }}$ (including amplitude and phase) can be measured. To make the short-circuit current 
$\boldsymbol{I}_{\text {sc }}$ large enough for measurement, the primary current was set to $\boldsymbol{I}_{\mathbf{p}}=100 \mathrm{~A} \angle 0^{\circ}$ in the experiment. Thus, the current coefficient $\boldsymbol{k}$ (in phasor form) can be derived from $\boldsymbol{k}=N *$ $\boldsymbol{I}_{\mathbf{s c}} / \boldsymbol{I}_{\mathbf{p}}$, where $N$ is 600 for the adopted CT. The result of the current coefficient is $\boldsymbol{k} \approx 1 \angle 0^{\circ}$, as listed in Table 2 .

2) In Fig. 8(b), remove the primary current and add a test voltage source to the secondary side. Use the power analyzer to measure the active power $P_{\mathrm{A}}$ and reactive power $Q_{\mathrm{A}}$. The test voltage $V_{\text {test(peak) }}$ was set to $60 \mathrm{~V}$, which is in the middle of the core linear region. From $P_{\mathrm{A}}, Q_{\mathrm{A}}$ and $V_{\text {test(RMS), }}$ the equivalent inductance $L_{\text {eq }}$ and equivalent resistance $R_{\text {eq }}$ can be derived according to (31) and (32). The results of the adopted CT are $L_{\text {eq }}=460 \mathrm{mH}$ and $R_{\text {eq }}=3.65 \mathrm{k} \Omega$, as listed in Table 2.

$$
\begin{gathered}
L_{\mathrm{eq}}=\frac{V_{\text {test(RMS })}^{2}}{\omega Q_{\mathrm{A}}} \\
R_{\mathrm{eq}}=\frac{V_{\text {test(RMS })}^{2}}{P_{\mathrm{A}}}
\end{gathered}
$$

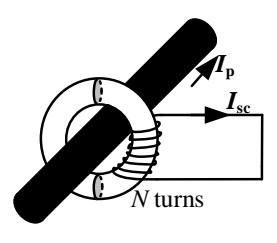

(a)

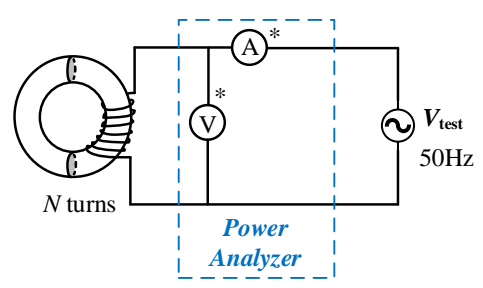

(b)
Fig. 8. Measurement setups of CT Norton parameters (a) Setup of measuring short-circuit current, (b) Setup of adding test voltage source

Besides, the critical saturation voltage $V_{\mathrm{CT}(\mathrm{sat})}$ also can be measured by the setup shown in Fig. 8(b). Since the core saturation will result in a dramatical decrease of $L_{\mathrm{eq}}$, we can obtain the saturation voltage by measuring the change of $L_{\mathrm{eq}}$ when the test voltage increases. In the experiment, the peak value of $V_{\text {test }}$ was increased from $20 \mathrm{~V}$ to $135 \mathrm{~V}$. The values of $L_{\text {eq }}$ were measured and depicted in Fig. 9. The dramatical decrease of $L_{\text {eq }}$ can be seen when the test voltage increases to a certain level. The criterion to define the critical saturation voltage in our design is the $1.5 \%$ decrease point compared to the linear region inductance. Since the value of $L_{\mathrm{eq}}$ in the linear region is around $460 \mathrm{mH}$, the critical saturation voltage $V_{\mathrm{CT}(\mathrm{sat})}$ is determined as $115 \mathrm{~V}$, where $L_{\mathrm{eq}}$ is $453.5 \mathrm{mH}$ (with $1.4 \%$ decrease compared to $460 \mathrm{mH}$ ).

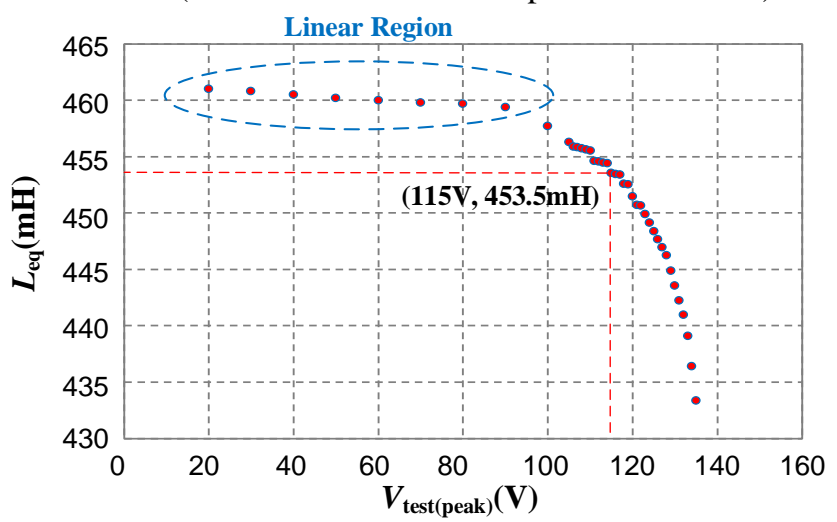

Fig. 9. Experimental results of $L_{e q}$ versus $V_{\text {test(peak) }}$

The experimental comparison between the CT with and without compensation was conducted, and the results are shown in Fig. 10(a) and (b). The rectifier was replaced by the sliding rheostat to achieve the input resistance change. The experimental results well track the theoretical calculation (8) and (12), except the compensated case with $30 \mathrm{~A}$. In the $20 \mathrm{~A}$ condition, the power increasing ratio by compensation is calculated as $1026 \mathrm{~mW} / 78 \mathrm{~mW} \approx 13$, which agrees with (14). For the compensated CT, the maximum power transfer is achieved at $3.64 \mathrm{k} \Omega$ load, which matches the CT parameter $R_{\text {eq }}$ in Table 2. In Fig. $10(\mathrm{~b})$, the experimental results of $30 \mathrm{~A}$ deviate from the theoretical calculation since the core enters saturation zone.

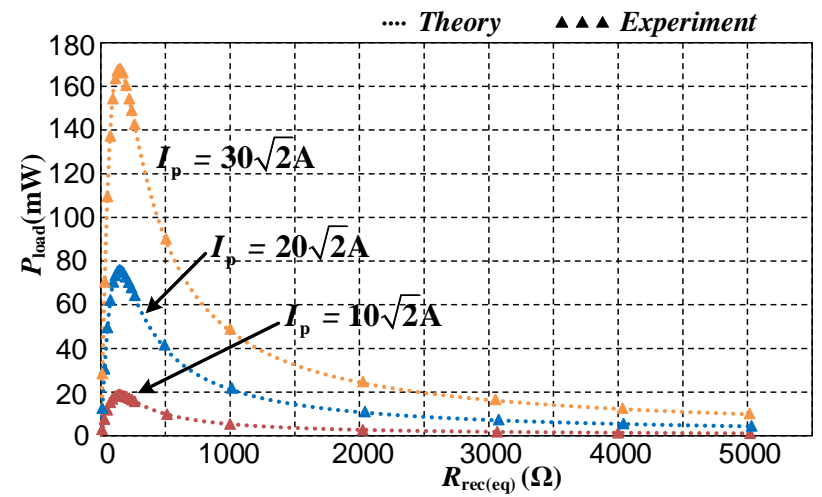

(a)

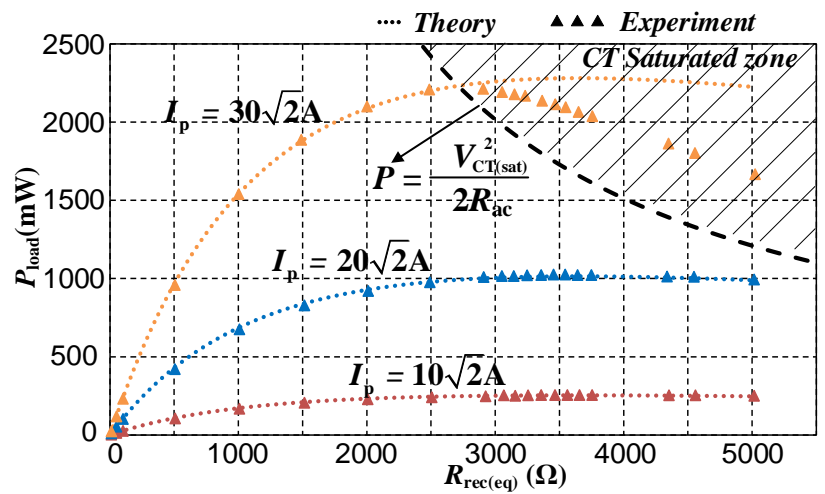

(b)

Fig. 10. CT output power: (a) Without compensation capacitor, (b) With compensation capacitor

For impedance matching, the buck-boost converter was controlled to work in DCM and the duty cycle was used to regulate the input resistance. Since the maximum power transfer is achieved when $R_{\text {rec(eq) }}=3.64 \mathrm{k} \Omega$, the buck-boost input resistance $R_{\text {con(eq) }}$ should be $7.28 \mathrm{k} \Omega$. The matching duty cycle $d_{\text {mpp }}$ can be calculated as 0.12 . The waveforms of the buck-boost converter working in impedance matching are shown in Fig. 11(a).

The short-circuit angle regulation was achieved by the $S$ CAR. The waveforms of short-circuit angle regulation are shown in Fig. 11(b). The relationship between CT output voltage and short-circuit angle was measured with $40 \mathrm{~A} \mathrm{rms}$ primary current and plotted in Fig. 12. The experimental results show a good consistency with the numerical results presented in Section 4 . The deviation of $4 \mathrm{k} \Omega$ curves at the beginning is due to the core saturation.

To implement the control strategy proposed in Section 5, two reference voltages were set as $V_{\mathrm{dc}(\text { low })}=103 \mathrm{~V}$ and $V_{\mathrm{dc}(\text { high })}=107 \mathrm{~V}$, and the coefficient $p_{\mathrm{d}}$ was set to 0.07 . The selections of the two reference voltages are mainly in consideration of 1) being close to the saturation voltage $V_{\mathrm{CT}(\mathrm{sat})}$ for power maximization; 2) ensuring the control stabilization. Specifically, $V_{\mathrm{dc}(\mathrm{high})}$ of $107 \mathrm{~V}$ is close to the 


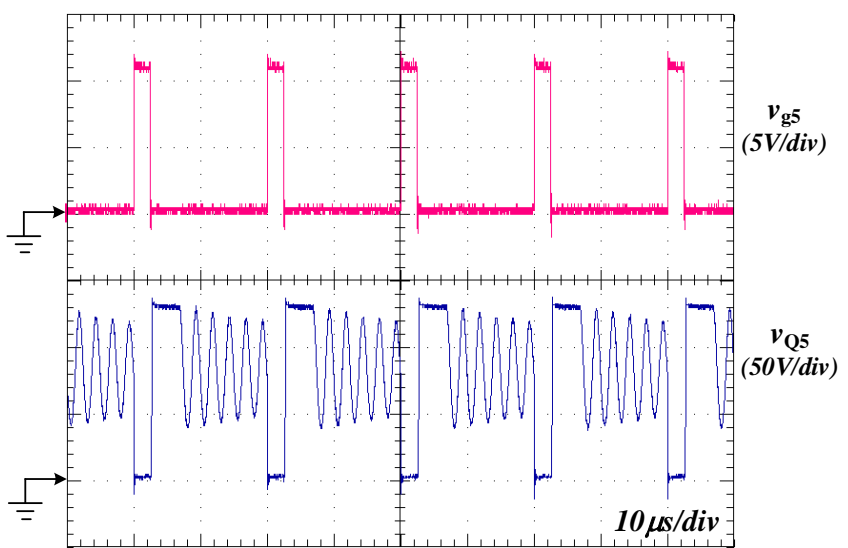

(a)

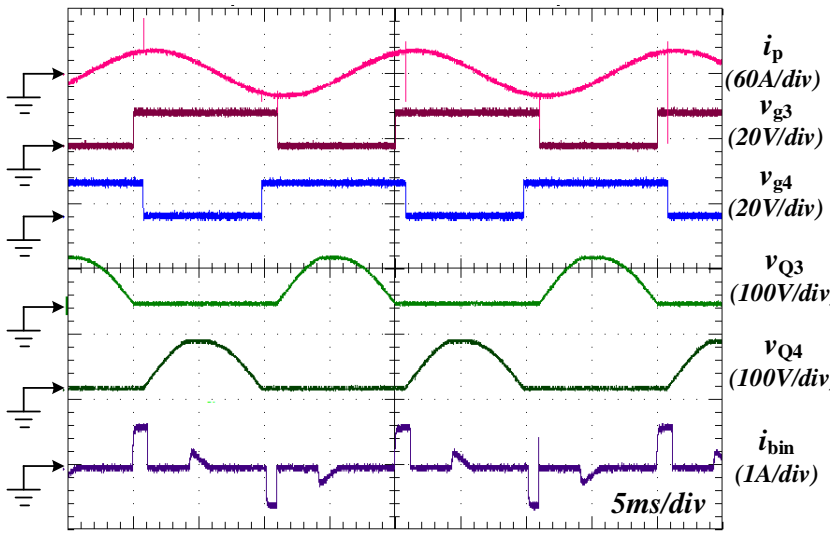

(b)

Fig. 11. Working Waveforms: (a) Buck-boost working in impedance matching, (b) S-CAR working with shortcircuit angle regulation

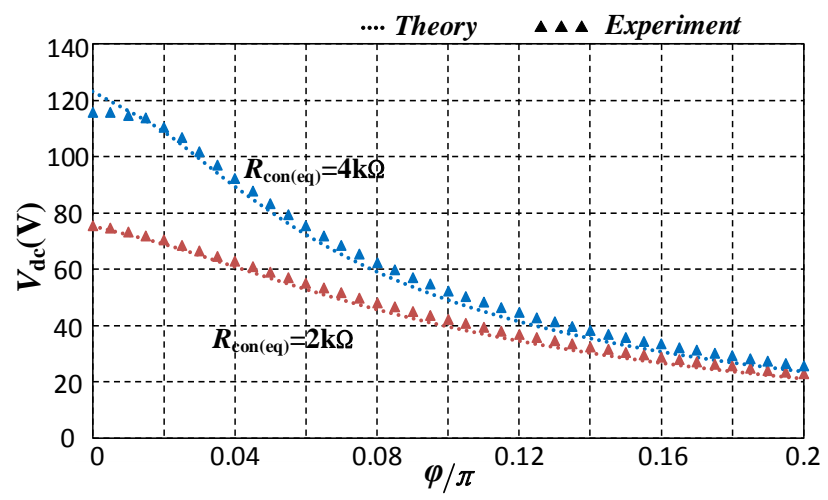

Fig. 12. Rectifier output voltage versus short-circuit angle

saturation voltage $115 \mathrm{~V}$ and has a margin of $8 \mathrm{~V}$ to avoid the deep saturation. $V_{\mathrm{dc}(\mathrm{low})}$ of $103 \mathrm{~V}$ was obtained by test, and the voltage gap of $4 \mathrm{~V}\left(V_{\mathrm{dc}(\text { high })}-V_{\mathrm{dc}(\text { low })}\right)$ proved enough to ensure the stabilization of the LOP state.

The harvester maximum output capability was measured and depicted in Fig. 13. The experimental results are consistent with the calculation of (30). Especially, the power density of magnetic core with $60 \mathrm{~A} \mathrm{rms}$ is $5520 \mathrm{~mW} / 120.1$ $\mathrm{cm}^{3}=45.96 \mathrm{~mW} / \mathrm{cm}^{3}$. The result in the same condition reported by [10] is $250 \mathrm{~mW} / 100.9 \mathrm{~cm}^{3}=2.48 \mathrm{~mW} / \mathrm{cm}^{3}$. Besides, the maximum output power with $200 \mathrm{~A} \mathrm{rms}$ is measured as $22.5 \mathrm{~W}$, which is 9 times higher than $2.5 \mathrm{~W}$ reported by [10]. A marked improvement is achieved by the prototype. The efficiency of the conditioning circuit is shown in Fig. 14. The efficiency is higher than $90 \%$ when the current exceeds $40 \mathrm{~A}$ rms. It should be noted that the power consumption of gate drive circuit and MCU was 0.15 $\mathrm{W}$, which was not counted in the efficiency calculation.

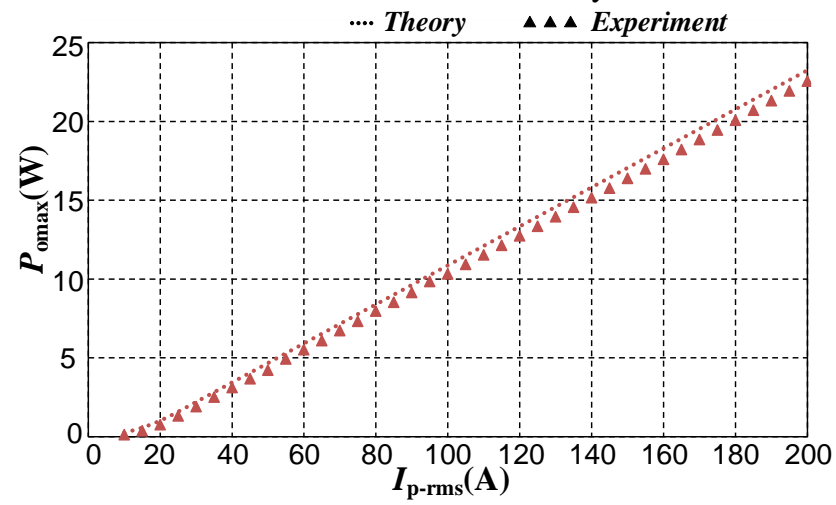

Fig. 13. Maximum output power versus primary current

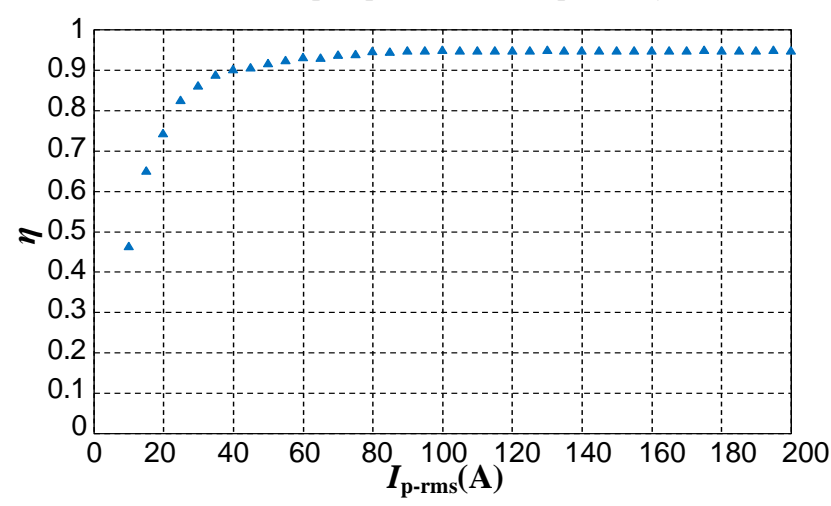

Fig. 14. Efficiency of the power conditioning circuit

Considering the real loads of CT harvester include sensors and communication devices, three samples listed in Table 4 are employed for the power evaluation. It can be seen that the power consumption of sensors can be neglected compared to the communication devices. Assume these devices in working mode, the total power consumption is $1798.5 \mathrm{~mW}$. On the other hand, $2 \mathrm{~W}$ can be harvested with $30 \mathrm{~A}$ as shown in Fig. 13. Thus, the startup current for independently powering the load can be set to $30 \mathrm{~A}(50 \mathrm{~Hz})$. The low startup current is beneficial to reduce the capacity of backup battery.

Table 4 Power consumptions of monitoring devices

\begin{tabular}{lll}
\hline \hline Function & \multicolumn{1}{c}{ Product } & \multicolumn{1}{c}{ Power Consumption } \\
\hline WLAN & NXP13201 Transceiver & Working: $90.5 \mathrm{~mW}$ \\
& & Idle: $1.35 \mathrm{~mW}$ \\
WWAN & HL7618 4G Module & Working: $1708 \mathrm{~mW}$ \\
& & Idle: $3.92 \mathrm{~mW}$ \\
Sensor & TI HDC1010 Sensor & $4.2 \mathrm{uW}$ (average) \\
\hline \hline
\end{tabular}

\section{Conclusion}

This paper proposes a power conditioning circuit with comprehensive control for the CT harvester on overhead power lines. The power maximization over the full current range is achieved by the coordination of compensation capacitor, impedance matching and short-circuit angle regulation. From a new viewpoint, the buck-boost converter is used for impedance matching to achieve CT maximum power transfer. The power maximization in low current condition is beneficial to reduce the capacity of backup battery. The short-circuit angle regulation achieved by $\mathrm{S}$ CAR is proposed to prevent the magnetic core from 
saturation. This method can make full use of the core linear region and will not result in extra self-dissipation like the dissipative resistor method.

A prototype that can deliver $22.5 \mathrm{~W}$ power at $200 \mathrm{~A}$ is designed. A control strategy based on the finite-state machine is implemented. The experimental results show that the startup current for $2 \mathrm{~W}$ load is about $30 \mathrm{~A}$, and the core power density at $60 \mathrm{~A}$ is $45.96 \mathrm{~mW} / \mathrm{cm}^{3}$, both of which are markedly improved compared to the reported results of the same condition.

\section{References}

[1] Greenwood, D.M., Gentle, J.P., Myers, K.S., et al.: 'A comparison of real-time thermal rating systems in the US and the UK', IEEE Trans. Power Del., 2014, 29, (4), pp. 1849-1858

[2] Savarimuthu, K., Sankararajan, R., Murugesan, S.: 'Analysis and design of power conditioning circuit for piezoelectric vibration energy harvester', IET Sci., Meas. Technol., 2017, 11, (6), pp. 723-730

[3] El-Hami, M., Glynne-Jones, P., White, N.M., et al.: 'Design and fabrication of a new vibration-based electromechanical power generator', Sens Actuators A, Phys., 2001, 92, (1), pp. 335-342

[4] Torres, E.O., Rincón-Mora, G.A.: 'Electrostatic energy-harvesting and battery-charging CMOS system prototype', IEEE Trans. Circuits Syst. I, Reg. Papers, 2009, 56, (9), pp. 1938-1948

[5] Zangl, H., Bretterklieber, T., Brasseur, G.: 'A feasibility study on autonomous online condition monitoring of high-voltage overhead power lines', IEEE Trans. Instrum. Meas., 2009, 58, (5), pp.17891796

[6] Moon, J., Leeb, S.B.: 'Power electronic circuits for magnetic energy harvesters', IEEE Trans. Power Electron., 2016, 31, (1), pp. 270-279

[7] Wu, Z., Wen, Y., Li, P.: 'A power supply of self-powered online monitoring systems for power cords', IEEE Trans. Energy Convers., 2013, 28, (4), pp. 921-928

[8] dos Santos, M.P., Vieira, D.A., Rodriguez, Y.P.M., et al.: 'Energy harvesting using magnetic induction considering different core materials', Proc. IEEE Int. Instrum. Meas. Technol. Conf., May 2014, pp. 942-944

[9] Li, P., Wen, Y., Zhang, Z., et al.: 'A high-efficiency management circuit using multiwinding upconversion current transformer for power-line energy harvesting', IEEE Trans. Ind. Electron., 2015, 62, (10), pp. 6327-6335
[10] Du, L., Wang, C., Li, X., et al.: 'A novel power supply of online monitoring systems for power transmission lines', IEEE Trans. Ind. Electron., 2010, 57, (8), pp. 2889-2895

[11] Wang, W., Huang, X., Tan, L., et al.: 'Optimization design of an inductive energy harvesting device for wireless power supply system overhead high-voltage power lines', Energies, 2016, 9, (4), pp. 242

[12] Bhuiyan, R.H., Dougal, R.A., Ali, M.: 'A miniature energy harvesting device for wireless sensors in electric power system', IEEE Sensors J., 2010, 10, (7), pp. 1249-1258

[13] Roscoe, N.M., Judd, M.D., Fitch, J.: 'Development of magnetic induction energy harvesting for condition monitoring', Proc. 44th Int. Univ. Power Eng. Conf., Sep. 2009, pp. 1-5

[14] Taithongchai, T., Leelarasmee, C.: 'Adaptive electromagnetic energy harvesting circuit for wireless sensor application', Proc. 6th Int. Conf. Electr. Eng./Electron. Comput. Telecommun. Inf. Technol., 2009, pp. 278-281

[15] 'Power Donut 3: PD3 instrumentation platform for overhead transmission lines (Jan. 2017) ', http://www.usi-power.com/powerdonut-line-monitor, accessed 10 January 2018

[16] 'Overhead transmission line monitoring system brochure (2014)', http://www.otlm.eu/brochure-documents, accessed 10 January 2018

[17] Roscoe, N.M., Judd, M.D.: 'Harvesting energy from magnetic fields to power condition monitoring sensors', IEEE Sensors J., 2013, 13, (6), pp. 2263-2270

[18] Yuan, S., Huang, Y., Zhou, J., et al.: 'Magnetic field energy harvesting under overhead power lines', IEEE Trans. Power Electron. 2015, 30, (11), pp. 6191-6202

[19] Carmo, J.P., Gonçalves, L.M., Correia, J.H.: 'Thermoelectric microconverter for energy harvesting systems', IEEE Trans. Ind. Electron., 2010, 57, (3), pp. 861-867

[20] Dondi, D., Bertacchini, A., Brunelli, D., et al.: 'Modeling and optimization of a solar energy harvester system for self-powered wireless sensor networks', IEEE Trans. Ind. Electron., 2008, 55, (7), pp. 2759-2766

[21] Tan, Y.K., Panda, S.K.: 'Optimized wind energy harvesting system using resistance emulator and active rectifier for wireless sensor nodes', IEEE Trans. Power Electron., 2011, 26, (1), pp. 38-50

[22] Lee, E.W.: 'Magnetostriction and magnetomechanical effects', Rep. Prog. Phys., 1955, 18, (1), pp. 184-229

[23] Li, Y., Liu, Q., Hu, S., et al.: 'A virtual impedance comprehensive control strategy for the controllably inductive power filtering system', IEEE Trans. Power Electron., 2017, 32, (2), pp. 920-926

[24] Wang, Y., Li, Y., Cao, Y., et al.: 'Hybrid AC/DC microgrid architecture with comprehensive control strategy for energy management of smart building', Int. J. Elect. Power Energy Syst., 2018, 101, pp. 151-161

\section{Appendices}

$$
\left\{\begin{array}{l}
i_{\mathrm{Leq}}(\varphi / \omega)=A_{1} \sin \varphi_{1}-\frac{k I_{\mathrm{p}} R_{\mathrm{eq}}}{N \omega L_{\mathrm{eq}}} \cos (\varphi+\delta)=-I_{\mathrm{Leq} 0} \\
v_{\mathrm{CT}}(\varphi / \omega)=L_{\mathrm{eq}} A_{1}\left(\omega \cos \varphi_{1}-\alpha_{1} \sin \varphi_{1}\right)+\frac{k I_{\mathrm{p}} R_{\mathrm{eq}}}{N} \sin (\varphi+\delta)=0 \\
v_{\mathrm{CT}}((\varphi+\alpha) / \omega)=L_{\mathrm{eq}} A_{1} e^{-\alpha_{1} \frac{\alpha}{\omega}}\left[\omega \cos \left(\alpha+\varphi_{1}\right)-\alpha_{1} \sin \left(\alpha+\varphi_{1}\right)\right]+\frac{k I_{\mathrm{p}} R_{\mathrm{eq}}}{N} \sin (\varphi+\alpha+\delta)=V_{\mathrm{dc}} \\
i_{\mathrm{Leq}}((\varphi+\alpha+\theta) / \omega)=A_{2} \sin \varphi_{2}-\frac{k I_{\mathrm{p}} R_{\mathrm{eq}}}{N \omega L_{\mathrm{eq}}} \cos (\varphi+\alpha+\theta+\delta)=A_{1} e^{-\alpha_{1} \frac{\alpha}{\omega}} \sin \left(\alpha+\varphi_{1}\right)-\frac{k I_{\mathrm{p}} R_{\mathrm{eq}}}{N \omega L_{\mathrm{eq}}} \cos (\varphi+\alpha+\delta)+\frac{\theta}{\omega L_{\mathrm{eq}}} V_{\mathrm{dc}} \\
v_{\mathrm{CT}}((\varphi+\alpha+\theta) / \omega)=L_{\mathrm{eq}} A_{2}\left(\omega \cos \varphi_{2}-\alpha_{1} \sin \varphi_{2}\right)+\frac{k I_{\mathrm{p}} R_{\mathrm{eq}}}{N} \sin (\varphi+\alpha+\theta+\delta)=V_{\mathrm{dc}} \\
i_{\mathrm{bin}}((\varphi+\alpha+\theta) / \omega)=\frac{k I_{\mathrm{p}}}{N} \sin (\varphi+\alpha+\theta+\delta)-\left[A_{1} e^{-\alpha_{1} \frac{\alpha}{\omega}} \sin \left(\alpha+\varphi_{1}\right)-\frac{k I_{\mathrm{p}} R_{\mathrm{eq}}}{N \omega L_{\mathrm{eq}}} \cos (\varphi+\alpha+\delta)+\frac{\theta}{\omega L_{\mathrm{eq}}} V_{\mathrm{dc}}\right]-\frac{V_{\mathrm{dc}}}{R_{\mathrm{eq}}}=0 \\
i_{\mathrm{Leq}}(\pi / \omega)=-A_{2} e^{-\alpha_{1} \frac{\pi-(\varphi+\alpha+\theta)}{\omega}} \sin \left[\varphi_{2}-(\varphi+\alpha+\theta)\right]+\frac{k I_{\mathrm{p}} R_{\mathrm{eq}}}{N \omega L_{\mathrm{eq}}} \cos \delta=I_{\mathrm{Leq} 0} \\
v_{\mathrm{CT}}(\pi / \omega)=L_{\mathrm{eq}} A_{2} e^{-\alpha_{1} \frac{\pi-(\varphi+\alpha+\theta)}{\omega}}\left\{-\omega \cos \left[\varphi_{2}-(\varphi+\alpha+\theta)\right]+\alpha_{1} \sin \left[\varphi_{2}-(\varphi+\alpha+\theta)\right]\right\}-\frac{k I_{\mathrm{p}} R_{\mathrm{eq}}}{N} \sin \delta=0 \\
\frac{\omega}{\pi} \int_{0}^{\pi / \omega} i_{\mathrm{bout}}(t) d t=\frac{\omega}{\pi} \int_{(\varphi+\alpha) / \omega}^{(\varphi+\alpha+\theta) / \omega}\left\{\frac{k I_{\mathrm{p}}}{N} \sin (\omega t+\delta)-\left[i_{\mathrm{Leq}}((\varphi+\alpha) / \omega)+\frac{V_{\mathrm{dc}}}{L_{\mathrm{eq}}}\left(t-\frac{\varphi+\alpha}{\omega}\right)\right]-\frac{V_{\mathrm{dc}}}{R_{\mathrm{eq}}}\right\} d t=\frac{V_{\mathrm{dc}}}{R_{\mathrm{dc}}}
\end{array}\right.
$$

